\title{
Bariatric Surgery in Management of Type 2 Diabetes
}

A. At-Alwan'1.

Tasgow Caledonian University, Health and Life Sciences, Glasgow, United Kingdom.

Type 2 diabetes is increasing globally in parallel to the increase of obesity, which is the major risk factor for type 2 diabetes. Through chronic low-grade inflammation; obesity leads to insulin resistance and thereby, type 2 diabetes. The 2 epidemics (obesity and diabetes) referred to them as one term which is "diabesity".

Bariatric surgery, which is intended to manage obesity, has demonstrated a dramatic impact on diabetes remission as well, which was commonly seen early after the operation and before attaining significant weight loss.

Bariatric surgery can be divided into 3 types: restrictive, malabsorptive and mixed procedures. Malabsorptive procedures are associated with higher diabetes remission and weight loss than restrictive procedures. On the other hand, the complication rates were also more prevalent after malabsorptive surgery.

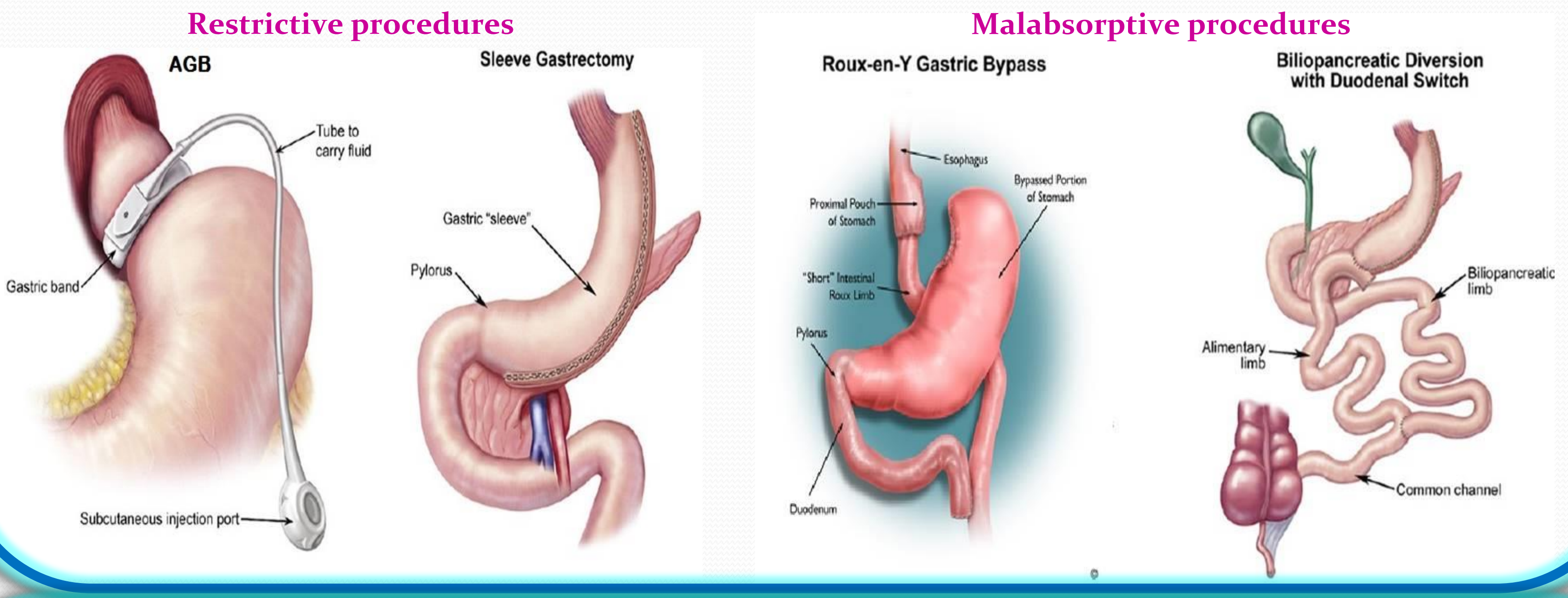

The mechanisms of remission were either weight lossdependant or weight loss-independent. The weight lossindependent mechanisms are mainly due to the hormonal changes. Some of the theories propose that rapid nutrients' delivery to the distal intestine triggers the release of incretin hormones, which has insulinotropic actions. Another theory suggests the nutrients' exclusion from the proximal intestine where anti-incretin factors are released. These two theories are implicated in improved glycaemia.

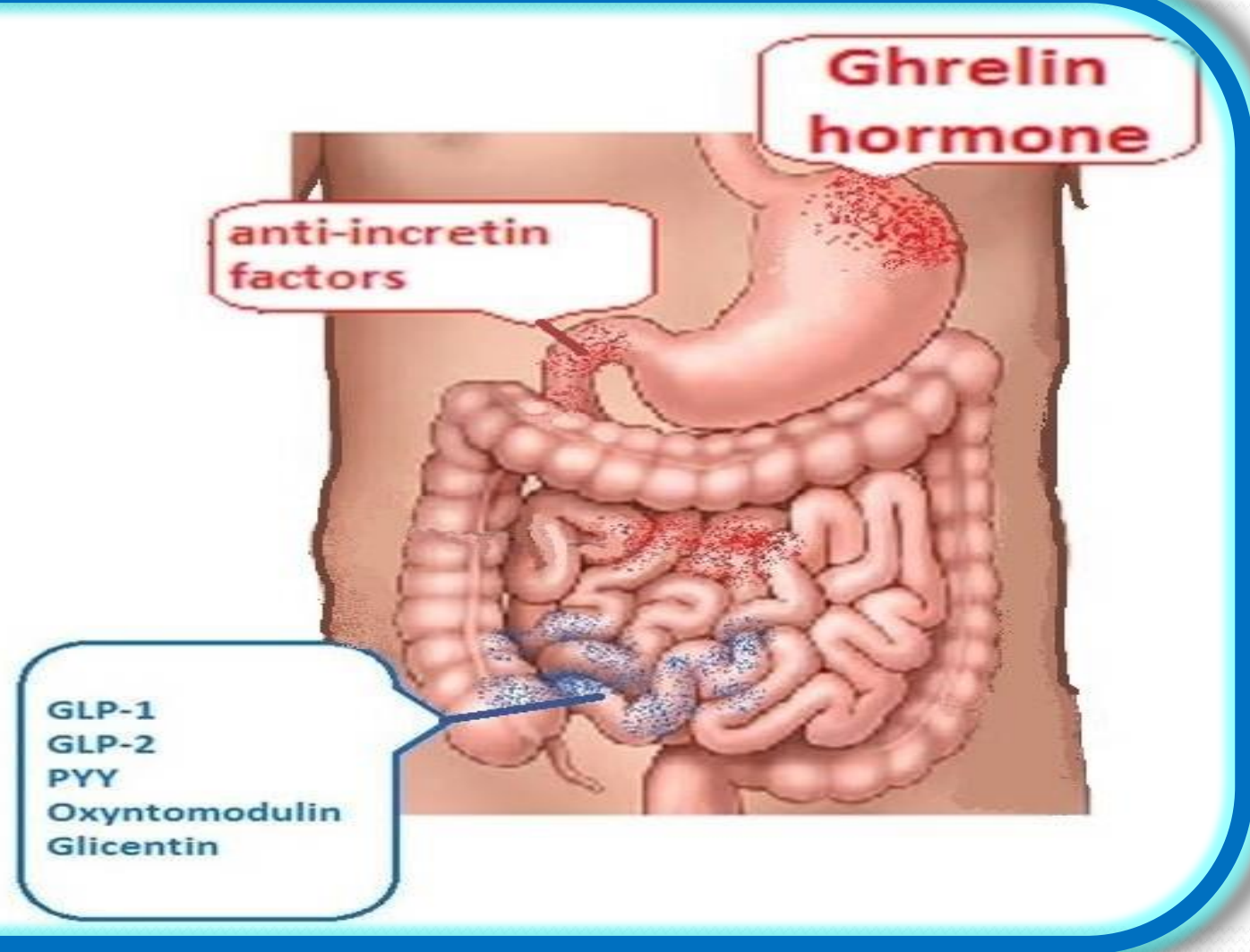

Some of the identified predictors of diabetes remission from the baseline characteristics are: higher body mass index, shorter duration of diabetes, controlled diabetes and younger age.

Bariatric surgery is honoured to be an effective option for treatment of diabesity. Nevertheless, benefits and risks should be weighed before making the choice of surgery.

\section{GCU}

Glasgow Caledonian University 